\title{
Self-localized structures in vertical-cavity surface-emitting lasers with external feedback
}

\author{
P. V. Paulau, ${ }^{1,2}$ D. Gomila, ${ }^{3}$ T. Ackemann, ${ }^{2}$ N. A. Loiko, ${ }^{1}$ and W. J. Firth ${ }^{2}$ \\ ${ }^{1}$ Institute of Physics, NASB, Belarus, Scaryna Prospekt 70, 220072 Minsk, Belarus \\ ${ }^{2}$ Department of Physics, University of Strathclyde, 107 Rottenrow East, Glasgow G4 ONG, United Kingdom \\ ${ }^{3}$ IFISC, Instituto de Física Interdisciplinar y Sistemas Complejos (CSIC-UIB), Campus Universitat Illes Balears, \\ E-07071 Palma de Mallorca, Spain
}

(Received 14 May 2008; published 28 July 2008)

\begin{abstract}
In this paper, we analyze a model of broad area vertical-cavity surface-emitting lasers subjected to frequency-selective optical feedback. In particular, we analyze the spatio-temporal regimes arising above threshold and the existence and dynamical properties of cavity solitons. We build the bifurcation diagram of stationary self-localized states, finding that branches of cavity solitons emerge from the degenerate Hopf bifurcations marking the homogeneous solutions with maximal and minimal gain. These branches collide in a saddle-node bifurcation, defining a maximum pump current for soliton existence that lies below the threshold of the laser without feedback. The properties of these cavity solitons are in good agreement with those observed in recent experiments.
\end{abstract}

DOI: 10.1103/PhysRevE.78.016212

PACS number(s): 42.65.Tg, 42.81.Dp

\section{INTRODUCTION}

Since design efforts in recent years have significantly improved the performance of vertical-cavity surface-emitting lasers (VCSELs), their applications in lightwave communications systems and for optical information processing are now being seriously explored. The most important attributes of VCSELs include a low threshold current, singlelongitudinal-mode operation, and direct compatibility with optical fiber waveguides. One of their specific features is connected with the possibility of creating devices with very broad area. Due to this, VCSELs can be considered as a paradigm for investigations of self-organized transverse structures in lasers, which could lead to novel applications.

In that respect, localized structures in VCSELs are especially attractive. They belong to the class of dissipative solitons, and more specifically to the cavity solitons (CS) family extensively studied in optics (see, e.g., [1-4]). Due to the requirement for energy balance in the cavity, their main characteristics have discrete values, ensuring their robustness. Particularly interesting features are bistability and mobility, which make them promising for future optical communication technologies. Laser CS, in contrast to CS in resonators with external driving $[4,5]$, do not require a monochromatic input beam of high spatial and temporal coherence, and are thus more attractive for applications. Cavity solitons were predicted first in lasers with a saturable absorber [6]. Laser CS based on a VCSEL with frequency-selective feedback, recently observed in experiment $[7,8]$, offer some extra advantages. For example, the external optical feedback allows monitoring and control of many characteristics of the compound system, and is also a potentially useful tool for study of the internal properties of VCSELs [8].

Some related theoretical considerations were studied in [9]. It was shown that bistability between lasing and nonlasing states, inherent to small-area VCSELs with frequencyselective feedback [10], is also observable in broad area devices with an additional spatial filter in the feedback loop. In that case, the dominant lasing state was a transverse traveling wave and, correspondingly and interestingly, the system displayed optical dissipative solitons consisting of self-localized traveling waves, i.e., CS at finite transverse wave numbers. Here we present theoretical results for systems without such spatial filtering, thus closer to the experimental situation $[7,8]$ where a system without spatial filtering yielded CS with the far field centered on axis. In the present work, we use a somewhat modified feedback model, and operate in a regime where limitation of spatial bandwidth arises as a natural consequence of the phase-amplitude coupling inherent in semiconductor lasers. We find and trace out the existence domain and bifurcation structure of on-axis CS. Our results are in qualitative agreement with the experimental observations and so can be useful (together with [9]) for the modeling of broad area lasers with different kinds of external feedback.

The paper is organized as follows: In Sec. II, we present our system model. In Sec. III, we discuss the stability of the nonlasing solution, and in Sec. IV we discuss the spatiotemporal regimes emerging from its instabilities. In Sec. V, we study the existence and stability properties of the localized solutions. Finally, in Sec. VI, we give some concluding remarks.

\section{SYSTEM AND MODEL}

In the experimental system $[7,8]$, which acts as motivation for our model, a broad area VCSEL is coupled to an external cavity formed by an afocal telescope and closed by a diffraction grating in Littrow configuration, which provides frequency filtering. We do not, however, attempt a detailed model of this grating-feedback system. Instead, with a view to finding and investigating behaviors that might have a rather general applicability, we study a simplified model depicted in Fig. 1, where the frequency selection is performed by a Lorentzian filter [11-13]. Such a description should also be approximately valid for a diffraction grating, and indeed for any frequency selective element with a single dominant feedback maximum (for example, a volume Bragg reflector). 


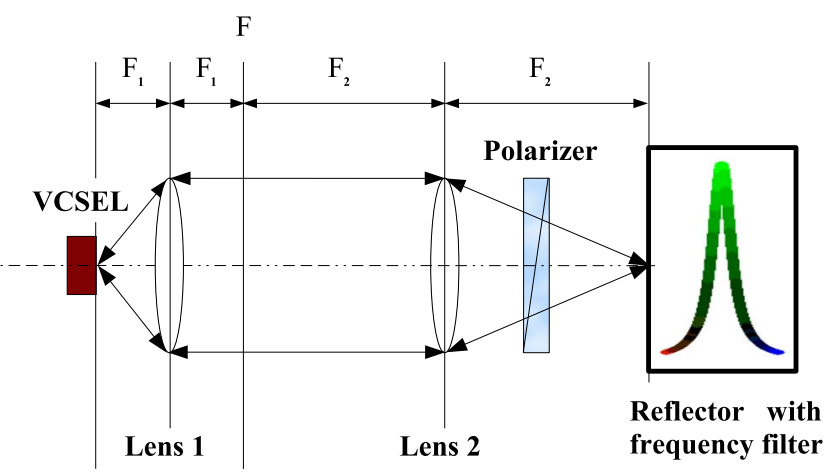

FIG. 1. (Color online) Scheme of the system. $F_{1}$ and $F_{2}$ are the focal lengths of the first and second lenses, respectively. The polarizer provides feedback only for the field component that is dominant in the solitary laser, so that the field can be treated as a scalar.

The carrier dynamics is adiabatically eliminated, since it does not influence the stationary states which are the main subject of this work. Moreover, we neglect all internal VCSEL wave-number selection mechanisms such as frequencydependent losses or gain [14]. This assumption is reasonable in regimes where lasing is possible only with external feedback, and where the bandwidth of that feedback is much less than the gain bandwidth. Nor do we allow for any angular dependence of the feedback spectrum or efficiency. Note also that our model does not take thermal effects into account, which implies that the system is kept at a fixed temperature. Due to the large number of simplifications, our model is not intended to provide a quantitative description of any particular experiment, but may nonetheless capture the general mechanisms underlying the formation of CS in VCSEL with frequency-selective feedback.

We describe the laser in terms of the slowly varying amplitude $E$ of the electric field $\mathcal{E}=E e^{i \omega_{0} t}+E^{*} e^{-i \omega_{0} t}$ (where $\omega_{0}$ is a reference frequency, chosen to be that of the filter maximum). Note that we use the same convention for positive frequency as in [9] and much of the optical feedback literature (e.g., [12]). Much of the CS literature uses the opposite convention.

Including all the approximations mentioned above, the rate equations for $E$ and for the feedback field $F$ are

$$
\begin{gathered}
\frac{\partial E}{\partial t}=-\kappa E+\frac{\kappa \mu E}{1+|E|^{2}}+\frac{i \alpha \kappa \mu E}{1+|E|^{2}}-i \Delta_{\perp} E+F+i \omega_{s} E-i \alpha \kappa E \\
\frac{d F}{d t}=-\lambda F+\sigma \lambda E(t-\tau)
\end{gathered}
$$

where $\kappa$ is the decay rate of the field in the cavity, $\alpha$ is the linewidth enhancement factor describing phase-amplitude coupling, $\mu$ is the pump current normalized to be 1.0 at the threshold of the solitary laser, $\Delta_{\perp}=\frac{\partial^{2}}{\partial x^{2}}+\frac{\partial^{2}}{\partial y^{2}}$ is the transverse Laplacian describing diffraction, $\omega_{s}$ is the detuning of the threshold frequency of the axial mode of the solitary laser from the filter peak, $\sigma$ is the feedback strength, $\tau$ is the delay time in the feedback loop, and $\lambda$ is the bandwidth of the filter reflection.

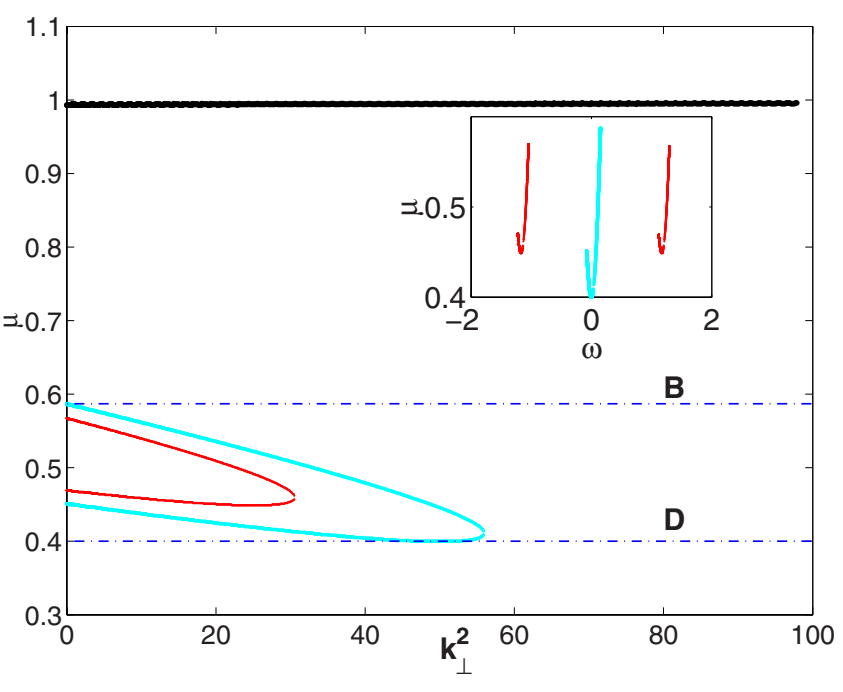

FIG. 2. (Color online) Marginal stability curves of the nonlasing state in the VCSEL with external feedback. The inset shows the frequencies of the modes whose stability boundaries lie between lines D and B in the main figure. The gray (cyan) line corresponds to the boundary of excitation of external cavity modes with frequencies close to $\omega=0$ (the filter maximum); the boundary of excitation of the modes on either side of the dominant mode, with frequencies $|\omega| \approx 1$ (these have very nearly equal thresholds for our parameters) follows the thin solid (red) line; the black bold line corresponds to the excitation of modes with frequencies close to those of the solitary laser. Parameters: $\sigma=60, \alpha=5.0, \lambda=2.71, \tau=5.0, \omega_{s}=250$, and $\kappa=100$. These parameters are kept fixed throughout the paper, and correspond physically to a time unit around $1 \mathrm{~ns}$.

Terms on the right-hand side of the $E$ equation describe, respectively, losses, which make our system dissipative; saturable gain, proportional to the pump current, where coefficient $\kappa$ is presented due to normalization of $\mu$ (see above); saturable Kerr-like nonlinearity, self-focusing for positive $\alpha$, which is typical for VCSELs; spatial coupling due to diffraction in the microcavity; and external feedback field. The last two terms are linear frequency shifts dependent on our choice of reference frequency.

\section{LINEAR STABILITY ANALYSIS}

As is typical in lasers without transverse degrees of freedom, the nonlasing state, or trivial solution $(E=0, F=0)$, loses its stability via a supercritical Hopf bifurcation on increasing the pump current. External feedback reduces the laser threshold, most strongly for modes with frequencies close to the filter reflection maximum. For suitably chosen values of the parameters, the range of pump values for which the trivial solution is unstable with respect to the excitation of external cavity modes may not overlap with the range of values for which it is unstable with respect to modes with frequencies close to those of a solitary laser [9]. The vertical axis of Fig. 2 illustrates this scenario for our model parameters, with the trivial (off) state being stable for $\mu$ in the range between line $\mathrm{B}$ and the black bold line in Fig. 2. Finite-amplitude solutions still exist in this gap, however, as can be seen by the obvious scaling of Eq. (1) with $\mu /(1$ 


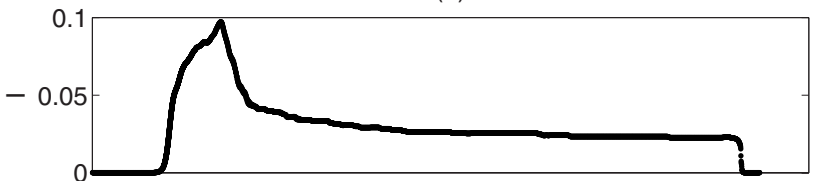

(b)

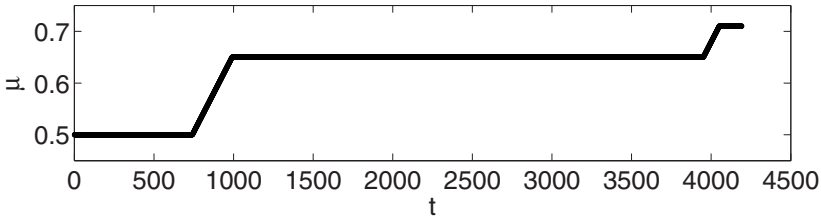

FIG. 3. (a) Time dependence of the integrated intensity $I$ $=\int_{-\infty}^{+\infty}|E(x, y)|^{2} d x d y$ for the sequence of pump current values shown in (b). The turn-off of the laser when the current is increased through $\mu=0.7$ is discussed and explained in Sec. V.

$+|E|^{2}$ ), which describes gain-clamping. In such a region, the trivial state can coexist stably with the lasing solution, providing optical bistability [10].

The transverse spatial degrees of freedom complicate the situation. Above threshold, a continuum of transverse Fourier modes can grow exponentially in the linear stage of the system evolution. For appropriate detunings $\omega_{s}$, the marginal stability curve typically looks like the one presented in Fig. 2 for $\omega_{s}=250$, where the trivial state is unstable to transverse perturbations between lines D and B as well above the bold black line. The gray (cyan) line is the marginal curve for the external cavity longitudinal mode with frequency closest to the grating maximum (see the inset of Fig. 2). The thin (red) line corresponds to the threshold of the adjacent modes (see also the inset of Fig. 2). The black line near $\mu=1.0$ arises due to the excitation of transverse modes with frequencies typical for the solitary laser. If the filter frequency is moved closer to the VCSEL resonance, the domain bounded by the gray (cyan) line is enlarged, and the interval of stability between line B and the bold black line is reduced and eventually disappears [9]. Conversely, if the filter is tuned further from the VCSEL resonance, the gray (cyan) and thin (red) marginal curves shift to the left, eventually disappearing. In that situation, feedback-controlled lasing no longer exists, and only solitary VCSEL modes (black bold line of in Fig. 2) remain.

\section{SPATIO-TEMPORAL REGIMES}

In this section, we perform numerical simulations for different pump currents. First we take $\mu=0.5$ (in the feedbacklasing region) and launch simulations starting from the nonlasing state with a small, random, spatially inhomogeneous initial field. All other parameters are indicated in the caption of Fig. 2 and kept fixed throughout this paper. The simulations show the total output growing exponentially out of the nonlasing state $[0<t<750$ in Fig. 3(a) corresponding to $\mu$ $=0.5$ in Fig. 3(b)] without settling to a constant value. Data for an extended run at constant $\mu=0.5$ show no sign of stabilization. Near and far fields shown in Figs. 4(a) and 4(b) show the developed regime to be spatially complex, as is the dynamics - the temporal evolution and optical spectrum are (a)

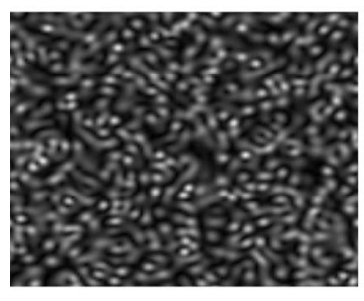

(c)

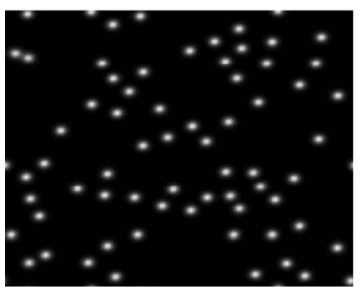

(b)

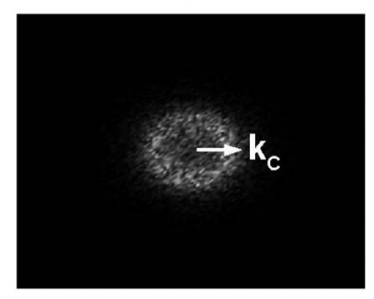

(d)

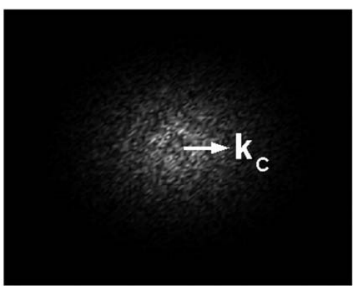

FIG. 4. Spatial distribution of amplitude of the (a) near and (b) far field for the spatio-temporal chaotic regime at $\mu=0.5$; (c) and (d) for the set of stationary solitons resulting from increasing the pump current to $\mu=0.65$. The white arrows in panels (b), (d) indicate the critical wave number [point of contact of line D and gray (cyan) curve in Fig. 2].

presented in Fig. 5. It can be seen from the near field that there is strong filamentation in the system [15], which can be attributed to the self-focusing nonlinearity $i \alpha \mu E /\left(1+|E|^{2}\right)$ in the first equation of Eqs. (1). In this regime, spots (i.e., filaments in 3D) continuously form, move, and decay in such a way that on average there is still a preferred length scale in the system. The far field shows excitation of a ring of transverse wave vectors, the modulus of which is in agreement with the stability analysis done in Sec. III (Fig. 2). In addition, the optical spectrum of the developed spatio-temporal

(a)
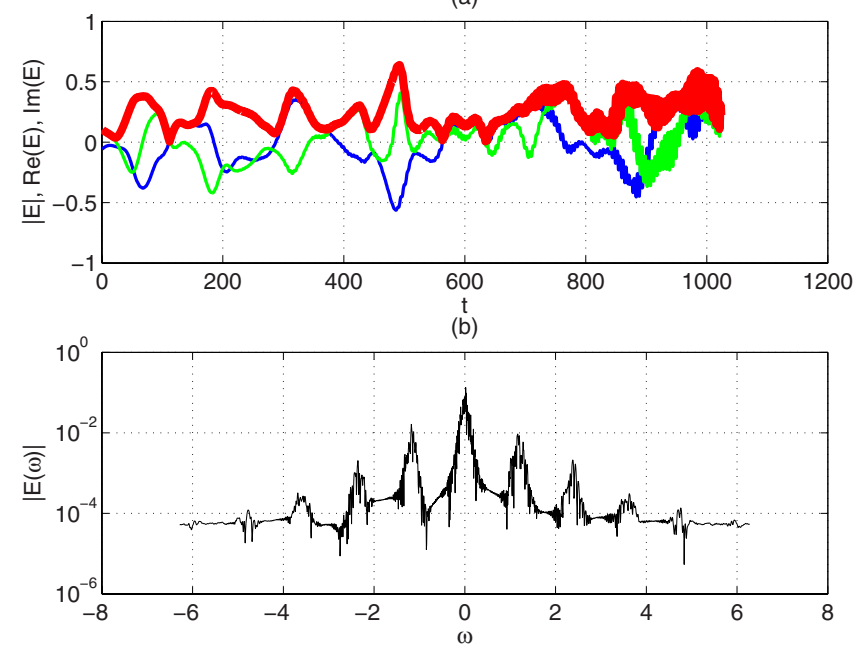

FIG. 5. (Color online) (a) Dynamics of the field at a selected point of the transverse section for the developed regime at $\mu=0.5$ (run for a longer time than in Fig. 3). Bold (red) line, amplitude of the field; thin gray (green) line, real part; thin black (blue) line, imaginary part of the field. (b) Optical spectrum of the time trace presented in (a) (Compare with the inset of Fig. 2.) 
regime shows pronounced modulation at the external-cavity mode separation [compare Fig. 5(b) and the inset of Fig. 2]. The thickening of the traces in Fig. 5(a) is due to this evolving multimode instability.

Departing from this irregular regime by slowly increasing the current $[750<t<1000$ in Fig. $3(\mathrm{~b})]$ to a value $\mu=0.65$ above line B in Fig. 2, we observe the spontaneous emergence of a set of stable self-localized structures (solitons) from the spatio-temporal chaos. In [16] a similar phenomenon was observed, but, instead of an irregular set of spots, a regular periodic pattern was reached. An intermediate behavior was observed in [17], where an Eckhaus unstable pattern "cracked" to form irregular patches of stable hexagonal pattern with dark voids in between. (There, as here, the dark state was stable.) Figures 4(c) and 4(d) show the spatial distribution of the near- and far-field amplitudes at $t=3800$, where the set of cavity solitons has stabilized and the integrated intensity is stationary. Repeating the simulations with different increment rates and initial field distributions, we find qualitatively the same behavior, though the number and positions of the solitons vary between runs. Our system, therefore, exhibits stable cavity solitons coexisting with a stable trivial background, but (in contrast to $[16,17]$ ) we have not observed bistability between any extended pattern and the background.

The irregular scatter of solitons persists indefinitely at constant current, but increasing the pump current above $\mu$ $=0.7$ ( $t$ around 4000 in Fig. 2), all the solitons disappear and the system falls back to the stable nonlasing state. The reason for this behavior will become clear in the next section.

The above-described method is an unusual way to excite cavity solitons, because on increasing a spatially homogeneous parameter (pump current), we have seen the spontaneous appearance of localized states. Here "spontaneous" means that solitons appear without applying any spatially localized perturbation, such as a "writing" or "address" beam. Spontaneous excitation is unexpected in systems where CS arise through "locking" of fronts between a homogeneous state and a subcritical pattern [18], but is observed experimentally in VCSELs with frequency-selective feedback [8], and in some other systems [3,19,20]. In the next section, we will find that the present CS have a very different bifurcation behavior from those previously studied.

\section{LOCALIZED SOLUTIONS}

To confirm the existence of localized states and to build their bifurcation diagram, we compute a single-peaked localized structure solutions using a Newton method $[21,22]$. We look for steady states of the form

$$
\begin{aligned}
& E=E_{0}(x, y) e^{i \omega t}, \\
& F=F_{0}(x, y) e^{i \omega t} .
\end{aligned}
$$

We call this a "steady state" because of its trivial dependence on time, which drops out from the equations due to the phase invariance in our system, allowing the Newton method to be
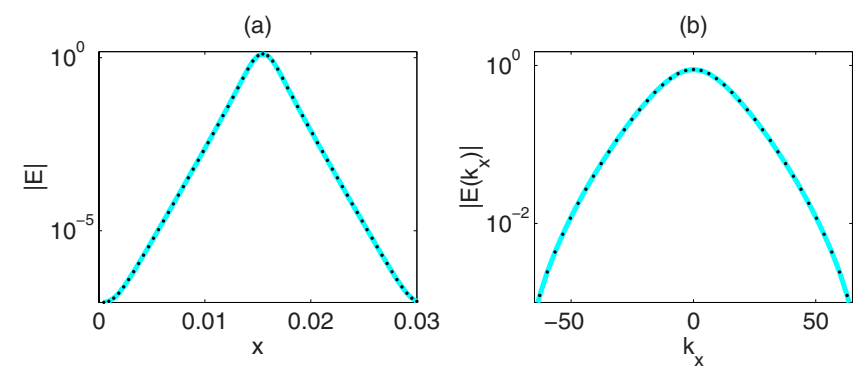

FIG. 6. (Color online) Section on a semilogarithmic scale of the near (a) and far (b) fields of a cavity soliton. Gray (cyan) line: direct integration of Eq. (1). Black points: Newton method. Here $\mu$ $=0.65$.

applied in the spatial domain. The lasing frequency $\omega$ is an additional, at present undetermined, parameter. We substitute Eq. (2) into Eq. (1) and derive a quite simple equation for the steady-state distribution $E_{0}(x, y)$,

$$
A E_{0}+B \frac{E_{0}}{1+\left|E_{0}\right|^{2}}+\Delta_{\perp} E_{0}=0,
$$

where the following auxiliary coefficients are introduced:

$$
\begin{gathered}
A=\omega-\omega_{s}+\kappa \alpha-i \kappa+\frac{\lambda \sigma(\omega+i \lambda)}{\lambda^{2}+\omega^{2}} e^{-i \omega \tau}, \\
B=i \kappa \mu(1+i \alpha) .
\end{gathered}
$$

Note that the state of our system is characterized by the spatial distribution $E_{0}(x, y)$ and the frequency $\omega$. The field distribution $E_{0}(x, y)$ is discretized: $E_{0}\left(x_{k}, y_{l}\right)$, where $k$ $=1, \ldots, N, l=1, \ldots, N$ with $N$ the number of grid points in each direction. The state of the system is then defined by $N \times N$ complex variables and one real variable. While Eq. (3) gives only $N \times N$ coupled complex equations, our system has an arbitrary global phase, which we choose to fix with an integral condition $[22,23]$ that provides the necessary additional equation.

Starting from an initial guess based on the solitonic states found by direct integration of Eqs. (1), we solve the highdimensional system of nonlinear equations resulting from discretizing Eq. (3) using a Newton-Raphson method (see [24]). We use linear algebra libraries for parallel architectures SCALAPACK [25] and calculate the transverse Laplacian in Fourier space using fftw libraries [26]. Figure 6 shows the near and far fields of a CS computed in this way. The linear slope in Fig. 6(a) indicates an exponential decay of the tails, thus showing the strong localization expected of a soliton. A qualitative difference between our localized states and those observed in externally driven cavities (see [5,21]) is the absence of oscillatory tails in the near field or, equivalently, of a ring around the central lobe in the far field. This feature is a consequence of the phase invariance in our system, which means that the trivial state has only one pair of stable or unstable eigenvalues, not two pairs as in these other systems. Hence the decay of both amplitude and phase of the soliton is smooth, as in Fig. 6. This is in agreement with the experimental results reported in [8], where no discernible ripples 

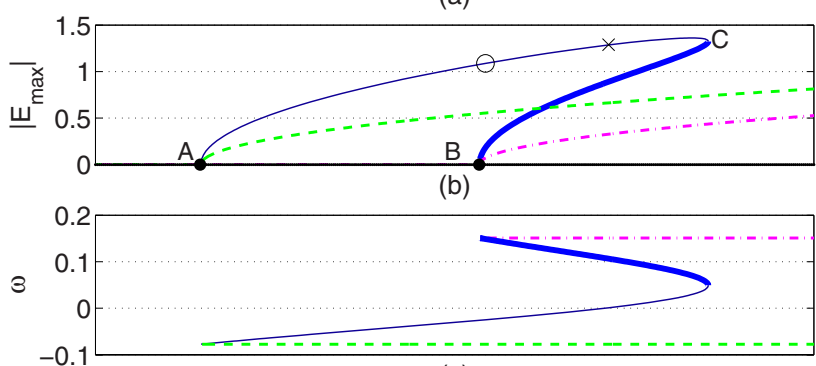

(c)

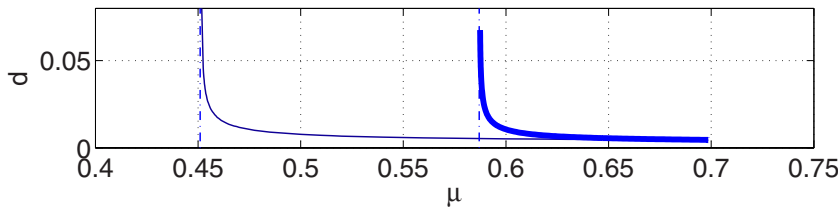

FIG. 7. (Color online) Dependence of (a) maximum field amplitude, (b) frequency, and (c) width of cavity solitons on pump current. Solid thin line "upper," thick line "lower" branches of CS. Dashed (dash-dotted) line in figures (a), (b) corresponds to the homogeneous external cavity mode with maximal (minimal) gain. The vertical lines in figure (c) correspond to the asymptotes at bifurcation points. Points A,B,C are explained in the text.

are found in the profiles of either near- or far-field intensity distributions. Note also that the computed CS are centered around zero wave number, as (within experimental resolution) are the experimental ones [8].

Continuing the solutions by varying $\mu$, we built the bifurcation diagram for CS solutions presented in Fig. 7. (The grid spacing is automatically adjusted to account for the variation of the CS width as the control parameter is changed.)

There are three bifurcation points in Fig. 7. At A and B, branches of CS solutions appear on increasing the pump. These bifurcation points are also present in the system without spatial dependence but they become degenerate when the spatial degrees of freedom are added. The third bifurcation point is saddle-node $\mathrm{C}$, where the two CS branches collide and annihilate. Beyond C, then, CS no longer exist, in agreement with the simulation results in Fig. 3 of Sec. IV.

The CS computed near A, B have very large width, consistent with asymptoting to infinite width at the bifurcation points. Width divergence at $A, B$ is consistent with the fact that the CS emerge from bifurcation points where nontrivial homogeneous solutions appear (see Figs. 2 and 7). On increasing the pump current, however, the FWHM rapidly decreases, as shown in Fig. 7(c). This can be interpreted as a self-focusing effect associated with an increase of the central intensity of the CS, and of its associated amplitude curvature. Note also that the increase of current is accompanied by a frequency shift [Fig. 7(b)], positive for the "upper" branch and negative for the "lower" one.

To fully assess the stability of CS, we would have to solve the characteristic equation of the linearized system. In the usual models, this stability problem is a straightforward eigenvalue problem, but the present case is a delay-differential system, and the characteristic quasipolynomial has an infinite number of roots. The stability problem is therefore much

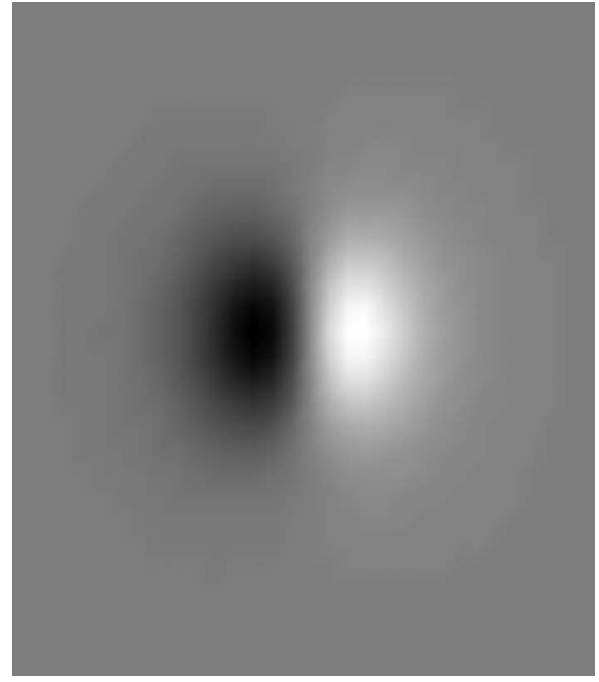

FIG. 8. Spatial structure of the most unstable mode of a driftunstable CS lying between the circle and cross in Fig. 7.

more complicated (see, for example, [27]). Therefore, we have so far only checked CS stability by direct integration of Eq. (1) at representative points on the CS existence curve.

Beginning with the upper branch, we find that if the initial point lies in the pump range where the trivial background is unstable, i.e., between points A and B in Fig. 7(a), the system goes to a spatio-temporal chaotic regime similar to Figs. 4(a) and 4(b). Between B and C, the trivial background is stable. We find that the CS are stable between point $\mathrm{C}$ and the cross. Note that the stable stationary CS all have positive frequencies, i.e., are blueshifted with respect to the filter frequency. This is in agreement with the recent experimental observations $[7,8]$. The size of the CS in the stable area is about $13 \mu \mathrm{m}$, in good agreement with the experimental finding of about $13 \mu \mathrm{m}$ [8].

At the upper-branch point marked with a cross, the CS develop a motional instability: stable constant-speed drifting CS are found between the cross and the circle. In Fig. 8, we plot the spatial structure of the most unstable mode in the drift-instability regime. It has been computed by starting a simulation from the drift-unstable stationary CS found using the Newton method, and calculating the field change after a small time interval. This mode strongly resembles the translational neutral mode, i.e., the gradient of the CS, as found in similar cases of drift instability, e.g., [28].

The lower solitonic branch (BC) is found to be always unstable. It acts as a separatrix between the basins of attraction of the trivial solution and the upper solitonic branch. That is, starting from a lower branch CS with upwardly perturbed amplitude, there is a transition to the upper branch: with amplitude perturbed downwards, the CS collapses into the stable background.

We note that the CS bifurcation structure in this system consists of just two branches (indeed a single folded branch). There is no sign of the homoclinic snaking found in other systems, such as those with a subcritical modulational instability [18], or those with bistability between homogeneous solutions [29]. In both of these cases, the snaking is associated with locking of front due to the presence of oscillatory 
tails. Our system does not exhibit such tails because of the phase symmetry, so the absence of snaking is perhaps to be expected.

\section{CONCLUDING REMARKS}

We have presented and analyzed a model of broad area VCSELs with frequency-selective external feedback. There is good qualitative agreement between our theoretically obtained solitons and the localized structures recently observed experimentally in a system of this kind $[7,8]$. For reasonable values of our parameters, there is also semiquantitative agreement, in that the widths of our CS are also in good accord with the experiments.

Our results also show the existence of stable moving localized structures, which appear as a result of a drift instability of the stationary CS. Typical speeds are quite small (about 40 ns for a CS to move a distance equal to its diameter), consistent with ignoring the carrier dynamics. Unlike systems where the motion of CS is caused by an imposed transverse inhomogeneity, for instance an inclination of the holding beam $[5,30,31]$, the motion in our system arises via a spontaneous breaking of the transverse translational symmetry, similar to, e.g., [28]. In contrast to motion due to Galilean invariance [2], where the CS can have arbitrary velocity, in our case the CS seem to have a fixed speed for fixed parameter values.

The CS branches we have obtained form a single folded loop, connected to Hopf bifurcation points of the homogeneous external cavity modes. This bifurcation structure shows no sign of the homoclinic snaking found in other systems. We ascribe this to the absence from our CS of oscillatory tails, because of the phase symmetry. However, the full CS bifurcation structure, and the question of existence of other families of localized states, in models like the present one needs much more investigation, and will be the subject of future studies.

To build on and extend the present study, we will obviously have to remove, or at least quantify, some of the approximations and simplifications that we made in the present work. For example, an accurate study of temporal regimes will need to account for carrier dynamics, including diffusion. We should also take into account angular variation of the feedback efficiency, which can introduce transverse forces acting on the solitons [8]. On the other hand, the novel and interesting characteristics of the cavity solitons found in the present model invite investigations into still simpler and more general models displaying these characteristics.

\section{ACKNOWLEDGMENTS}

P.V.P. was supported by INTAS Grant No. 04-83-3276 and by BRFFI Grant No. Ф06M-053. The work of T.A. and W.J.F. was carried out in the framework of EU-STREP No. 4868 FunFACS. D.G. acknowledge financial support from the Spanish Ministry of Education (MEC) and Govern Balear under Grant No. TEC2006-10009 and PROGECIB$5 \mathrm{~A}$. We are grateful to R. Martin for help with the use of parallel libraries, and to A. Scroggie and A. Naumenko for useful discussions.
[1] Dissipative Solitons, edited by N. Akhmediev and A. Ankiewicz (Springer, New York, 2005).

[2] N. N. Rosanov, Spatial Hysteresis and Optical Patterns, Springer Series in Synergetics (Springer, Berlin, 2002).

[3] C. O. Weiss and Ye Larionova, Rep. Prog. Phys. 70, 255 (2007).

[4] X. Hachair, F. Pedaci, E. Caboche, S. Barland, M. Giudici, J. R. Tredicce, F. Prati, G. Tissoni, R. Kheradmand, L. A. Lugiato, I. Protsenko, and M. Brambilla, IEEE J. Sel. Top. Quantum Electron. 12, 339 (2006).

[5] W. J. Firth and A. J. Scroggie, Phys. Rev. Lett. 76, 1623 (1996).

[6] N. N. Rosanov and S. V. Fedorov, Opt. Spectrosc. 72, 1394 (1992).

[7] Y. Tanguy, T. Ackemann, and R. Jäger, Phys. Rev. A 74, 053824 (2006).

[8] Y. Tanguy, T. Ackemann, W. J. Firth, and R. Jäger, Phys. Rev. Lett. 100, 013907 (2008).

[9] P. V. Paulau, A. J. Scroggie, A. Naumenko, T. Ackemann, N. A. Loiko, and W. J. Firth, Phys. Rev. E 75, 056208 (2007).

[10] A. Naumenko, N. Loiko, M. Sondermann, K. Jentsch, and T. Ackemann, Opt. Commun. 259/2, 823 (2006).

[11] M. Guidici, L. Giuggioli, C. Green, and J. R. Tredicce, Chaos, Solitons Fractals 10, 811 (1999).

[12] M. Yousefi and D. Lenstra, IEEE J. Quantum Electron. 35,
970 (1999).

[13] A. P. A. Fisher, O. K. Andersen, M. Yousefi, S. Stolte, and D. Lenstra, IEEE J. Quantum Electron. 36, 375 (2000).

[14] N. A. Loiko and I. V. Babushkin, J. Opt. B: Quantum Semiclassical Opt. 3, S234 (2001).

[15] I. Fischer, O. Hess, W. Elsäßer, and E. Gobel, Europhys. Lett. 35, 579 (1996); J. V. Moloney, J. Opt. B: Quantum Semiclassical Opt. 1, 183 (1999).

[16] D. Gomila and P. Colet, Phys. Rev. A 68, 011801(R) (2003); Phys. Rev. E 76, 016217 (2007).

[17] J. M. McSloy, W. J. Firth, G. K. Harkness, and G.-L. Oppo, Phys. Rev. E 66, 046606 (2002).

[18] P. D. Woods and A. R. Champneys, Physica D 129, 147 (1999); P. Coullet, C. Riera, and C. Tresser, Phys. Rev. Lett. 84, 3069 (2000).

[19] V. B. Taranenko, F. J. Ahlers, and K. Pierz, Appl. Phys. B Lasers Opt. 75, 75 (2002).

[20] Y. Menesguen, S. Barbay, X. Hachair, L. Leroy, I. Sagnes, and R. Kuszelewicz, Phys. Rev. A 74, 023818 (2006).

[21] W. J. Firth, G. K. Harkness, A. Lord, J. M. McSloy, D. Gomila, and P. Colet, J. Opt. Soc. Am. B 19, 747 (2002).

[22] A. J. Scroggie, D. Gomila, W. J. Firth, and G.-L Oppo, Appl. Phys. B: Lasers Opt. 81, 963 (2005).

[23] Y. A. Kuznetsov, Elements of Applied Bifurcation Theory, 2nd ed. (Springer, New York, 1998). 
[24] W. H. Press, S. A. Teukolsky, W. T. Vetterling, and B. P. Flannery, Numerical Recipes in C The Art of Scientific Computing (Cambridge University Press, Cambridge, 1997).

[25] J. Choi, J. Dongarra, S. Ostrouchov, A. Petitet, D. Walker, and R. C. Whaley, LAPACK, Working Note 100, A proposal for a Set of Parallel Basic Linear Algebra Subprograms, Computer Science Dept. Technical Report CS-95-292, University of Tennessee, Knoxville, July, 1995.

[26] M. Frigo and S. G. Johnson, FFTW User's Manual (http:// www.fftw.org/).

[27] K. Engelborghs, T. Luzyanina, and G. Samaey, DDE-Biftool v.2.00 User Manual: A Matlab package for bifurcation analysis of delay differential equations, Technical Report TW-330, Department of Computer Science, K. U. Leuven, Leuven, Belgium, 2001.

[28] A. J. Scroggie, J. M. McSloy, and W. J. Firth, Phys. Rev. E 66, 036607 (2002).

[29] J. Knobloch and T. Wagenknecht, Physica D 206, 82 (2005).

[30] O. Egorov, F. Lederer, and K. Staliunas, Opt. Lett. 32, 2106 (2007).

[31] O. Egorov, U. Peschel, and F. Lederer, Phys. Rev. E 72, 066603 (2005). 